\title{
L'essor de la pratique clinique dans les armées européennes (1750-1800) *
}

Othmar Keel et Philippe Hudon

\section{Summary}

This article demonstrates that, before the 19th century and the Paris Clinical School, new medical practice and new clinical teaching based on pathological anatomy (of organs and of tissues) and surgical experience and therapeutic experimentation developed in the military milieu, specifically because of the "auspicious" conditions found there. Over time, this military clinical experience permeated civilian medical practice as military practitioners often moved into civilian practice and collaborated and exchanged experience with their civilian or ex-military colleagues. These conditions, in different forms and at different rhythms, in the great European powers, also favoured a rapprochement between the different groups of medical practitioners physicians, apothecaries and surgeons - initially in the military milieu, and subsequently in civilian society as well. Finally, the article shows that the coercive disciplinary structure of the military, where sick or wounded soldiers were particularly constrained to act as subjects of experience, experimentation, clinical teaching and anatomico-pathological research, was one of the conditions propitious to this growth of clinical practice.

* Ce travail a bénéficié d'une subvention du Conseil de Recherche en Sciences Humaines du Canada. Les auteurs remercient Madame le Dr Andrée Yanacopoulo pour ses très utiles commentaires.

Othmar Keel (Professeur titulaire), Philippe Hudon (Doctorant), Département d'Histoire, Université de Montréal, C.P. 6128, succursale Centre-ville, Montréal, Québec, CANADA $\mathrm{H} 3 \mathrm{C} 3 \mathrm{~J} 7$ 


\section{Résumé}

Cet article montre que c'est antérieurement au $19^{e}$ siècle et à l'École clinique de Paris que l'on voit se développer, en raison de conditions propices dans le milieu militaire et, par suite, dans le milieu civil, une nouvelle pratique et un nouvel enseignement cliniques basés sur l'anatomie pathologiques (des organes et des tissus), sur l'expérience chirurgicale et sur l'expérimentation thérapeutique. Ces conditions favorisent aussi dans les différentes grandes puissances européennes, sous des formes et à des rythmes différents, un rapprochement entre les divers corps de praticiens: médecins, chirurgiens, pharmaciens dans le milieu militaire et aussi, par effet d'entraînement, dans le milieu civil.L'article montre enfin que l'une des conditions qui ont favorisé cet essor de la pratique clinique dans le milieu militaire est le cadre coercitif et disciplinaire propre à ce milieu, où les soldats malades ou blessés sont particulièrement astreints à servir de sujets d'expérience, d'expérimentation, d'enseignement clinique et de recherche anatomopathologique.

Au cours du $18^{\mathrm{e}}$ siècle, les armées des grandes puissances européennes, soit la France, la Prusse, l'Autriche et l'Angleterre, sont dotées de services de santé, organismes médico-militaires chargés de veiller au soin des soldats ${ }^{1}$. Le rôle des services de santé des armées est très diversifié et important. Ils

$1 \mathrm{Il}$ existe plusieurs grandes études sur l'état de santé des soldats et l'état des connaissances médicales dans les armées de certaines grandes puissances européennes pendant les $18^{\mathrm{e}}$ et $19^{\mathrm{e}}$ siècles. En ce qui a trait aux armées françaises, mentionnons les ouvrages de Raoul Brice et du capitaine Bottet, Le corps de santé militaire en France. Son évolution, ses campagnes (1708-1882), Paris et Nancy, 1907; Jean des Cilleuls, J. Hassenforder, J. Pesme, G. Hugonot, Le service de santé militaire de ses origines à nos jours, Paris, 1961; Pierre Huard, Mirko D. Grmek, Sciences, médecine, pharmacie de la Révolution à l'Empire (1789-1815), Paris, 1970; David M. Vess, Medical Revolution in France, 1789-1796, Gainesville (Florida), 1975; et les travaux du Comité d'histoire du Service de santé, Histoire de la médecine aux armées, Paris et Limoges, tome 1er: «De l'Antiquité à la Révolution», Jean Guillermand (dir.), 1982; tome 2: «De la Révolution française au conflit mondial de 1914», Albert Fabre (dir.), 1984; Monique Lucenet, Les problèmes de santé dans l'armée de terre française au $18^{\circ}$ siècle, Paris, 1986, thèse d'Histoire (Paris IV-Sorbonne), 3 vol. Pour une introduction à la question de la médecine au sein des armées allemandes, consulter Rudolf Bruppacher, Militärmedizin in der Aufklärung, Zürich, 1967. Pour les armées autrichiennes, voir Erna Lesky, Meilensteine der Wiener Medizin. Grosse Ärzte Österreichs in drei Jahrhunderten, Wien, 1981, et Joachim Moerchel, Das österreichische Militärsanitätswesen im Zeitalter des aufgeklärten Absolutismus, Frankfurt, 1984. Sur les armées anglaises, se référer à Christopher Lloyd et Jack L. S. Coulter, Medicine and the Navy, 1200-1900, tome 3 (1714-1815), Edinburgh \& London, 1961; Neil Cantlie, A History of the Army Medical Department, Edinburgh \& London, 1974, 2 vol.; Paul E. Kopperman, «Medical Services in the British Army, 1742-1783» dans Journal of the History of Medicine and Allied Sciences, vol. 34, n ${ }^{\circ} .4,1979$, pp. 428-455. Pour la Prusse et l'Europe en général, consulter Fielding H. Garrison, Notes on the History of Military Medicine, Hildesheim, 1922, et New York, 1970. 
sont organisés pour répondre aux missions qui leur incomberont lorsque les pays auxquels ils appartiennent entreront en guerre, à savoir principalement la gestion des hôpitaux par des administrateurs, le maintien de la police et de la propreté des formations sanitaires, confiés à des commissaires, et la prestation de soins médico-chirurgicaux, assurés par les officiers de santé et leurs aides. En France, par exemple, ces fonctions sont parfaitement séparées et précisées de façon à éviter, autant que possible, des conflits entre ces trois rouages administratifs ${ }^{2}$. Parallèlement, les services forment, par le truchement de l'enseignement théorique, pratique et clinique, ou par l'expérience, un très grand nombre de nouveaux praticiens qui vont fournir un apport important aux connaissances médicales générales.

Considérant les dangers que peut provoquer l'ensemble des maladies qui affectent les armées, les services de santé éviteront au maximum la contamination de leurs soldats qui, de plus, se propage très souvent au milieu civil. Malgré les difficultés, parfois gigantesques, contre lesquels ils doivent lutter ${ }^{3}$, les services de santé des armées européennes sont appelés à jouer un rôle de plus en plus important à une époque qui est témoin de l'intensification des politiques nationales de santé. La médecine acquiert une place et un intérêt de plus en plus marqués aux yeux des gouvernements et des états-majors ${ }^{4}$. Cette transformation donne lieu à ce que Foucault nomme une «machinerie de pouvoir» ${ }^{5}$ qui s'étend et s'affirme plus spécialement dans le milieu militaire. Ainsi, les services de santé des armées s'intègrent lentement mais sûrement à ce nouveau dispositif biopolitique pour devenir les exécutants des enquêtes médico-militaires qui visent à supprimer la mauvaise hygiène et les

$2 C f$. Huard et Grmek, Sciences, p. 45.

3 Pour les autorités politiques ou militaires, le Service de santé demeure essentiellement un «service» soumis à leurs ordres. Les corps médicaux sont de surcroît tenus de rendre compte de l'assurance et de la qualité de leurs prestations aux supérieurs: intendants, inspecteurs et commissaires. Les décisions concernant la marche des hôpitaux relèvent toujours de ces derniers et les services en souffrent parfois. $C f$. Hubert Bourgeois, article «Santé (Service de santé militaire)» dans Jean Tulard (dir.), Dictionnaire Napoléon, Paris, 1987, pp. 1530-1531. D'ailleurs, leur qualité dépend toujours de l'efficacité de l'administration des armées et des possibilités de leur financement. Voir à ce sujet André Corvisier, article «Santé» dans André Corvisier (dir.), Dictionnaire d'art et d'histoire militaires, Paris, 1988, p. 765; Guillermand, Histoire, tome $1^{\text {er }}$, pp. $458-460$.

4 Voir Garrison, Notes, pp. 162-163. En 1788, le Service de santé des armées françaises comprend trois inspecteurs (un médecin, un chirurgien et un pharmacien), 86 médecins des hôpitaux militaires, 192 chirurgiens-majors attachés aux régiments, 94 chirurgiens-majors affectés aux hôpitaux, 65 chirurgiens aides-majors, 38 chirurgiens sous-aides, 118 chirurgiens élèves dans les hôpitaux et 130 pharmaciens de tous grades, soit 726 membres dont 508 chirurgiens. $C f$. Guillermand, Histoire, tome $1^{\text {er }}$, p. 424. A la Révolution, les effectifs du Service de santé de ce même pays sont les suivants: 1400 en 1792, 2570 en 1793, 8000 en 1794 et 10000 en 1795. Huard et Grmek, Sciences, p. 43; Bourgeois, «Santé», p. 1530.

5 Michel Foucault, «La politique de la santé au $18^{e}$ siècle» dans Michel Foucault et al., Les machines à guérir. Aux origines de l'hôpital moderne, Bruxelles, 1979, pp. 7-18. 
grands fléaux (endémiques ou épidémiques) des troupes, et à identifier les soldats malades afin de les hospitaliser. Ces enquêtes doivent permettre le rapprochement des états-majors et des services de santé tout en garantissant l'augmentation sensible du pouvoir et du prestige de ces derniers. C'est dans ce contexte que l'on assiste à l'essor de la clinique entendue à la fois comme pratique, comme recherche et comme formation, ainsi que d'une pathologie anatomo-localiste dans le milieu militaire.

\section{La transformation des milieux hospitaliers}

La seconde moitié du $18^{\mathrm{e}}$ siècle voit la naissance et /ou le développement de services d'assistance médicale et d'hygiène dans une grande partie de l'Europe. C'est également le début de l'ère des réformes hospitalières. L'instauration de politiques nationales de santé conduit à la multiplication des praticiens en médecine, à la fondation et à la diversification des établissements hospitaliers ${ }^{6}$, et surtout à l'élargissement des soins prodigués à la fois chez les civils et chez les militaires. De même, les efforts pour améliorer la formation des médecins, des chirurgiens et des pharmaciens donnent de bons résultats ${ }^{7}$. Dans la pratique, la société finit par accorder une importance plus grande au développement des connaissances médicales ${ }^{8}$ et les patients, y compris les militaires, ont davantage confiance en la médecine?

A partir des années 1750, il y a une profonde transformation de l'infrastructure hospitalière des armées ${ }^{10}$. Elle s'observe dans toute l'Europe. Ce changement affecte à la fois les structures internes et externes des établissements hospitaliers. Parmi les exemples de la transformation des structures internes, mentionnons la séparation des malades, le réarrangement des

6 Par exemple,sur l'état général des hôpitaux français de l'Ancien Régime, voir Muriel Jeorger, «La structure hospitalière de la France sous l'Ancien Régime» dans Annales E. S. C., vol. 32, $\mathrm{n}^{\circ}$ 5, 1977, pp. 1025-1051.

7 Sur les effets de la politique de santé en Europe, cf. Foucault et al., Machines; Othmar Keel, Cabanis et la généalogie épistémologique de la médecine clinique, thèse de doctorat, McGill University, 1977, pp. 404 sq et tout le chapitre 10. Voir aussi Idem, «The Politics of Health and the Institutionalization of Clinical Practices in Europe in the Second Half of the Eighteenth Century», dans William F. Bynum et Roy Porter, William Hunter and the Eighteenth Century Medical World, Cambridge, 1985, pp. 207-256; ainsi que Thomas Neville Bonner, Becoming a Physician. Medical Education in Britain, France, Germany and the United States, 1750-1945, New York \& Oxford, 1995.

8 Foucault et al., Machines, p. 7; Irvine Loudon, Medical Care and the General Practitioner 1750-1850, Oxford, 1986.

9 Marie-José Imbault-Huart, «Les chirurgiens et l'esprit chirurgical en France au $18^{\mathrm{e}}$ siècle» dans Clio Medica, vol. 15, n 3/4,1981, pp. 143-157; Foucault et al., Machines.

10 Cette infrastructure comprend aussi bien les hôpitaux militaires maritimes que ceux pour les armées de terre. 
locaux, l'apparition de la clinique et la réorientation des méthodes d'enseignement. Notons surtout la redéfinition des rapports entre, d'une part, les praticiens des hôpitaux, et entre, d'autre part, le corps médical et ses patients. Les militaires hospitalisés sont mieux encadrés mais toujours soumis, comme simples soldats, à la double autorité des officiers de santé: autorité médicale et autorité militaire. Cette double discipline doit faire des soldats malades des patients plus dociles et plus observables que les civils, c'est-à-dire des sujets plus utiles pour la médecine ${ }^{11}$. La redéfinition en question porte également sur les rapports entre les praticiens et leur rôle en milieu hospitalier. On observe, notamment, un rapprochement de facto entre les médecins, les chirurgiens et les pharmaciens. Cette collaboration est rendue possible par la spécificité et l'uniformité des maladies rencontrées aux armées (blessés, fiévreux, galeux et vénériens qui forment plus de $90 \%$ des malades), par l'état de guerre, par le rôle accru du chirurgien et par l'insistance des décrets gouvernementaux.

Les transformations que nous qualifions d'«externes» ont trait, en particulier, à l'extension et à la diversification des réseaux nationaux des hôpitaux militaires. Il y a désormais des hôpitaux ambulants, des hôpitaux spéciaux, des hôpitaux d'instruction, etc. Ajoutons à ceci l'aménagement et la sélection, souvent fort rigoureuse, du cadre physique, l'immensité des établissements nouvellement construits ${ }^{12}$, l'instauration du système dit d'«hôpital

11 Un chirurgien militaire britannique, Robert Gordon, a publié en 1762 un ouvrage intitulé Regulations for Hospital Management, dans lequel il propose des mesures disciplinaires: «No man on any pretence of illness to be excused parade or any other duty, unless reported to the surgeon, and when reported the sergeant or corporal of his company is immediately to send him to hospital when taken ill; if unable to walk, he is to be carried. (...) If any man in hospital is guilty of irregularity, or refuses to comply with orders of the hospital-physician or sergeant, or makes any disturbance, or shall misbehave himself to the sergeant or his superior officer of the hospital, he will be severely punished». Cité dans Garrison, Notes, p. 155. Sur la discipline à la fois médicale et militaire dans les hôpitaux militaires de campagne en Grande-Bretagne, $c f$. Ibid., pp. 155-156.

12 Mentionnons par exemple l'hôpital militaire de Strasbourg, le plus grand hôpital militaire français de l'Ancien Régime. Terminé en 1742, il possède 1800 lits. Les hôpitaux de Metz et de Nancy, construits peu après, ont assez d'espace pour contenir près de mille lits chacun. En Autriche, on assiste à la création, à Vienne en 1785, de l'Académie Joséphine militaire médico-chirurgicale qui est en fait une école supérieure de chirurgie et de médecine équivalente à une université puisqu'elle confère un doctorat en chirurgie. A cette académie est rattaché le très vaste hôpital militaire (Garnisonspital) qui compte 1200 lits. Il est institué par l'Empereur Joseph II qui avait déjà fondé le très grand Allgemeines Krankenhaus (1784). La Grande-Bretagne possède aussi de gigantesques institutions médicales militaires qui ont servi de modèle pour la transformation des hôpitaux militaires et civils en Europe. Par exemple, l'hôpital naval royal de Haslar qui comprend 2000 lits en 1761, et l'hôpital royal de la marine à Plymouth qui en contient 1200. A ce sujet, $c f$. Keel, Cabanis, pp. 404 sq et tout le chapitre 10; Idem, «Politics», pp. 232-233 et pp. 242-246; Lesky, Meilensteine, pp. 29-53. Pour la mise en place des hôpitaux militaires en Prusse, voir Garrison, Notes, p. 141. Enfin, sur les établissements militaires britanniques de campagne et de la marine comme terrains de la 
mixte» et l'apparition d'hôpitaux spéciaux exclusivement réservés aux fiévreux, aux galeux, aux vénériens, etc. En temps de guerre, il arrive souvent que les militaires soient admis dans des hôpitaux civils. Ces établissements nommés «hôpitaux mixtes» hébergent à la fois des civils et des militaires, ce qui permet des échanges d'expérience, voire une coopération entre praticiens militaires et civils ${ }^{13}$. Il y a, d'autre part, des médecins civils qui exercent dans des hôpitaux militaires tout en gardant leurs prérogatives ${ }^{14}$. Exemple, l'Hôtel des Invalides: cet établissement a une infirmerie de 300 lits et une apothicairerie. Des chirurgiens, des médecins et des pharmaciens y travaillent; ils y possèdent un statut mi-civil, mi-militaire ${ }^{15}$.

Un autre type d'hôpital militaire apparaît à cette époque: l'hôpital «spécial», qui se distingue par la catégorie de patients qui y sont admis. On assiste, en effet, à la mise en place d'hôpitaux spéciaux ou de départements spécialisés (comme à l'hôpital d'Haslar) destinés à recevoir des blessés, des fiévreux ou encore des galeux et des vénériens. Ce genre d'établissement est bien davantage un centre de spécialisation de soins qu'une institution d'exclusion ou d'assistance. En fait, l'hôpital dit «unifonctionnel» apparaît au moment où l'hospitalisation devient un des piliers d'une politique de santé effective.

Plus spécifiquement, les hôpitaux militaires sont l'objet de réformes diverses qui répondent à certains besoins comme la valorisation de la santé des troupes ou la quantification de leurs besoins médicaux. Les armées apparaissent comme une force nationale très importante qui devient un objet d'étude médicale sans précédent. En France, ce type de politique de santé militaire prend sa forme sous le règne de Louis XIV avec, notamment, la multiplication des hôpitaux fixes ${ }^{16}$, la fondation de l'Hôtel royal des Invalides (1674) et la création du «service nosocomial militaire des frontières et places

pratique et de la recherche clinique, $c f$. Ulrich Troehler, Quantification in British Medicine and Surgery, 1750-1820, thèse de doctorat, University College of London, Londres, 1978, pp. 202 sq.

$13 C f$. la thèse de Lydie Boulle, Hôpitaux parisiens, malades et maladies à l'heure des révolutions (1789-1848), thèse de doctorat, Paris, 1986. Consulter plus spécifiquement le chapitre XI, «Militaires dans les hôpitaux et hospices civils».

14 Paul Delaunay, La vie médicale aux 16e, $17^{e}$ et $18^{e}$ siècles, Paris, 1935, p. 280.

15 Ibid., p. 280. Sur l'histoire de l'Hôtel royal des Invalides, $c f$. l'ouvrage du Musée de l'armée, Les Invalides. Trois siècles d'histoire, René Baillargeat (dir.), Paris, 1974.

16 On en compte 90 en 1781, ce nombre a doublé depuis 1708, date de l'instauration du Service de santé en France. Les hôpitaux fixes sont les plus grands et les plus importants des établissements hospitaliers des armées. Ils sont également nommés hôpitaux «permanents». Les hôpitaux fixes représentent sans aucun doute une des pierres angulaires des services de santé puisqu'ils doivent procurer un support sanitaire omniprésent (accueil des soldats blessés et malades, enseignement pratique et théorique, clinique, tenue de statistiques), déjà assuré à partir du milieu du siècle. Delaunay, Vie médicale, pp. 84-85. 
de guerre» $(1708)^{17}$. A partir des dernières décennies du $18^{\mathrm{e}}$ siècle, l'hôpital militaire devient plus fonctionnel, il couvre désormais l'espace militaire du pays, prend en charge les soldats malades, s'articule sur l'acquisition de nouvelles connaissances scientifiques et techniques, et sert d'appui à l'encadrement sanitaire des armées. Il a pour mandat d'accueillir de nombreux patients et de regrouper les services de santé afin de les rendre plus cohérents, plus faciles à contrôler et, bien sûr, moins coûteux. L'hôpital militaire doit être efficace et doit fonctionner idéalement comme une «machine à guérir» ${ }^{18}$. Il devient peu à peu un lieu hautement discipliné tout en demeurant un modèle d'utilité médicale ayant pour objectif ultime de faire progresser les sciences et les techniques par la tenue de statistiques, par l'expérimentation, par la clinique, par les examens et par de fréquentes visites ${ }^{19}$. Cette structure offre un modèle pour la médicalisation des populations en entier. La médicalisation des hôpitaux militaires a donc souvent de l'avance sur la médicalisation des établissements civils ${ }^{20}$. Foucault va même plus loin en affirmant que «la mise en ordre des hôpitaux maritimes et militaires sert de schéma à toute la réorganisation hospitalière du $18^{\mathrm{e}}$ siècle» ${ }^{21}$.

Néanmoins, on dénote un progrès inégal dans les applications de cette médecine nouvelle en Europe. En fait, les aspects et les conditions de la médicalisation varient selon les pays et selon les milieu ${ }^{22}$. Malgré ces développements d'ordre inégal, la médicalisation dans le milieu militaire, puis civil, finit par produire des résultats avec une observation des malades plus systématique, une expérience et un enseignement plus adéquat chez les praticiens. Et peu à peu la médecine dans son ensemble en bénéficie ${ }^{23}$. Par ailleurs, les dernières décennies du $18^{e}$ siècle verront se multiplier les inspections et les missions d'information dans les hôpitaux militaires. Elles dénonceront les abus, les négligences, les insuffisances et préconiseront des mesures susceptibles d'améliorer les conditions de vie et d'hospitalisation des malades. De

17 Ibid., p. 277.

18 Foucault et al., Machines, pp. 16 et 32.

19 Foucault écrit qu'«une des conditions essentielles pour le déblocage épistémologique de la médecine à la fin du $18^{\mathrm{e}}$ siècle fut l'organisation de l'hôpital comme appareil à examiner. Le rituel de la visite en est la forme la plus voyante... Peu à peu la visite est devenue plus régulière, plus rigoureuse, plus étendue surtout: elle a recouvert une part de plus en plus importante du fonctionnement hospitalier». Foucault, Surveiller et punir. Naissance de la prison, Paris, 1975, p. 187. Cf. également Keel, Cabanis, pp. 527 sq. En fait, l’organisation de l'hôpital comme appareil à examiner, particulièrement dans le milieu militaire, commence à se mettre en place dans plusieurs pays européens dès le milieu du $18^{\mathrm{e}}$ siècle.

20 Keel, «Politics», pp. 246-247; voir aussi Troehler, Quantification, pp. 429-438.

21 Foucault, Surveiller, p. 211.

22 Sur la médicalisation partielle et très inégale des hôpitaux européens au $18^{\mathrm{e}}$ siècle, voir Keel, «Politics»,pp. 220 sq.

23 Ibid., pp. 220 sq.; Cantlie, History, p. 170 sq. 
ce fait, les taux moyens de mortalité due aux grands fléaux des armées, par exemple, auront tendance à baisser ${ }^{24}$. En somme, les conditions de santé aux armées vont globalement, et parfois contre toute attente, s'améliorer grâce aux progrès des sciences et des techniques médicales, rendus possibles par la mise en place des politiques de santé nouvelles et par la médicalisation progressive des hôpitaux militaires.

\section{La redéfinition des rôles des praticiens militaires}

Dans les armées européennes du $18^{\mathrm{e}}$ siècle, les décisions relatives aux congés, à l'hospitalisation et à la sélection des malades admis relèvent des médecins et des chirurgiens. Par exemple, les chirurgiens prennent en charge les galeux et les vénériens car les premiers symptômes de leur maladie sont externes, cutanés, et peuvent faire l'objet d'une intervention directe. En outre, ils appliquent (ou font appliquer) les pommades, opèrent au niveau de la peau lésée, amputent si cela s'avère nécessaire, contrôlent les diètes et font administrer les médicaments. Du reste, les chirurgiens soignent la quasitotalité des blessés (bandages, amputations, débridements, etc.) et une grande partie des malades fiévreux.

Dans les armées françaises, par exemple, le chirurgien a le statut de chirurgien-major. Il détient, d'une part, le titre médical octroyé par son diplôme de maître en chirurgie après plusieurs années d'études; d'autre part, le grade de major fait de lui un officier supérieur. Le chirurgien-major, bien avant la Révolution, est devenu un personnage important et respecté ${ }^{25}$. Quant au médecin, qui a le titre de «médecin-major», distinction supérieure à celle de chirurgien-major, il a dans les services de santé un pouvoir important et de grandes responsabilités. Il a notamment la tâche traditionnelle de prescrire aux malades les purges et les saignées, d'ailleurs administrées par les chirurgiens et leurs aides. De plus, il lui incombe de soigner en particulier les soldats dits «fiévreux», ces patients atteints de maladies contagieuses diverses très fréquentes dans les campements et dont la pathologie est interne. A partir des règnes de Louis XIV et de Louis XV, le rôle des médecins militaires a

24 M. W. Flinn, The European Demographic System, 1500-1820, Brighton, 1981, pp. 95 sq. La plus grande discipline des armées et la nouvelle hygiène militaire sont aussi une des causes de la baisse de la mortalité de la population civile, plus souvent contaminée auparavant par les armées.

25 Avant la Révolution, il y a en moyenne, en France, un chirurgien-major et un médecin-enchef par hôpital permanent et par régiment. Voir par exemple Delaunay, Vie médicale, pp. 278 sq. Sur le statut et les fonctions des chirurgiens militaires en Allemagne et en Autriche, $c f$. respectivement Bruppacher, Militärmedizin, et Moerchel, Militärsanitätswesen. 
tendance à devenir de plus en plus important. Il s'accroîtra davantage encore dans les années $1760-1770{ }^{26}$

Les soldats malades sont généralement soignés par les médications qui leur sont spécifiquement réservées. Le choix des remèdes à administrer relève des chirurgiens-majors et des médecins-majors ou, parfois, des conseils de santé. Et celui de certains médicaments relève de l'autorité exclusive de $l^{\prime} \mathrm{Etat}^{27}$, qui tient ardemment à ses prérogatives en la matière. Cependant, le dosage et la préparation des remèdes relèvent surtout des apothicairesen-chef ou des pharmaciens-majors des armées. Ces derniers deviennent également des praticiens très importants aux côtés des médecins et des chirurgiens. En effet, leur concours fournit des résultats très intéressants de par leur expérience et leur pratique sur des sujets malades. Les pharmaciens militaires ont donc une participation très active dans le processus de médicalisation des armées, terrains propices aux expériences les plus diverses. $\mathrm{La}$ présence des trois corps de santé permet donc aux milieux médico-militaires de connaître les applications, dès le $18^{\mathrm{e}}$ siècle, d'une médecine clinique exercée sur des milliers de soldats malades.

Ce rapprochement des rôles des trois grandes professions médicales aux armées est facilité non seulement par l'état de guerre ou par la spécificité des pathologies rencontrées chez les soldats, mais aussi par la pratique, très fréquente, d'expériences thérapeutiques sur des patients qui leur servent de sujets. Cette pratique a même parfois lieu en concomitance avec le milieu civil. Ces expériences sont facilitées par le fait que les soldats malades sont des subalternes de chirurgiens, médecins et pharmaciens gradés. Les officiers de santé peuvent, après tout, exiger des soldats de se conformer aux expériences en question. Delaunay souligne l'ampleur de ces expériences thérapeutiques pour le cas des soldats vénériens, affirmant que «très fréquemment, les soldats malades en traitement dans les régiments ou dans les hôpitaux militaires servaient, par ordre du Ministre, de sujets d'expérience pour quelque traitement nouveau; les essais de spécifiques antivénériens pratiqués dans ces conditions au cours du $18^{\mathrm{e}}$ siècle sont particulièrement nombreux $»^{28}$. On assiste à une transformation des rôles médicaux favorisée par les pathologies

26 Voir Delaunay, Vie médicale, pp. 277 sq. On observe le même processus pour les médecins militaires en Allemagne, en Autriche et même en Angleterre. Sur leur statut dans ces pays, $c f$. Garrison, Notes.

27 C'est le cas, par exemple, des dragées de Keyser et des pilules de Belloste, deux médicaments employés contre les maladies vénériennes. $C f$. Philippe Hudon, La gale et les maladies vénériennes dans les armées françaises de la fin de l'Ancien Régime à la Révolution (1750-1800), mémoire de maîtrise en Histoire, Université de Montréal, 1990, pp. 130-132.

28 Paul Delaunay, Médecine militaire d'autrefois. L'École militaire de chirurgie renoueuse et la dynastie médicale des Valdajou, Lille, 1913, p. 66. Pour le cas spécifique des militaires galeux ou vénériens soumis à des expériences thérapeutiques diverses, $C f$. Hudon, La gale, pp. 
plus spécifiques aux armées. Chirurgiens, médecins et pharmaciens, longtemps séparés les uns des autres dans leur pratique médicale, finissent, malgré leurs rivalités de corps professionnels, par collaborer pour combattre les nombreux fléaux qui ravagent les troupes ${ }^{29}$. Les pharmaciens, chirurgiens et médecins militaires sont de plus en plus qualifiés et interviennent de concert dans le processus thérapeutique.

La nécessité d'une approche pluridisciplinaire des malades et de leurs pathologies témoigne, dans le milieu militaire comme dans le milieu civil, du souci d'améliorer leurs conditions de vie, mais également de mettre un frein à certaines pratiques déloyales, de vaincre la médiocrité et de contrer les méfaits des «charlatans» ${ }^{30}$. Soulignons à ce propos que l'Etat, qui possède une autorité quasi absolue sur l'administration des remèdes dans les hôpitaux militaires ${ }^{31}$, exerce un contrôle serré à ce niveau en faisant tester par des essais cliniques les nouveaux médicaments ${ }^{32}$.

\section{Importance accrue de l'enseignement et de la pratique clinique}

L'enseignement médical est aussi important aux armées que dans le milieu civil. En effet, les officiers de santé doivent acquérir une formation spécifique adaptée aux réalités militaires. Leur formation doit être pluridisciplinaire,

104-106. Le cas des soldats vénériens est applicable à toutes les catégories de malades aux armées. Lucenet nous dit que vers l'année 1776 on mène des expériences médicales sur 46 hommes stationnés à la citadelle de Metz, ainsi que sur des patients de l'hôpital militaire de Besançon. D'autres expériences ont lieu à Bicêtre et à Saint-Denis (Paris). Lucenet, Problèmes, p. 481.

29 Cf. Vess, Revolution, pp. 149-150. Sur le cas particulier de la gale et des maladies vénériennes, voir Hudon, La gale, pp. 102-104 et 106-116. Au 18 $8^{\mathrm{e}}$ siècle, on assiste dans la plupart des pays européens à un certain décloisonnement des rôles dans la pratique civile aussi bien que militaire. $C f$. Keel, Cabanis; Idem, «Les rapports entre médecine et chirurgie dans la grande école anglaise de William et John Hunter», dans Gesnerus, 45, 1988, pp. 323-341.

30 A ce sujet, lire Jean-Pierre Goubert, «L'art de guérir. Médecine savante et médecine populaire dans la France de 1790», dans Annales E. S. C., vol. 32, n 5, 1977, pp. 908-926. Cf. également François Lebrun, Se soigner autrefois. Médecins, saints et sorciers aux $17^{e}$ et $18^{e}$ siècles, Paris, 1983; Matthew Ramsey, Professional and Popular Medicine in France, 1770-1830, Cambridge, 1988.

31 Selon l'ordonnance royale de 1780 , pour «opposer une barrière aux manœuvres insidieuses des charlatans, des empiriques et des prétendus hommes à secret, Sa Majesté défend qu'aucun remède nouveau, interne ou externe, soit introduit dans ses hôpitaux, que préalablement le Secrétaire d'Etat de la guerre en ait fait reconnaître la nature et les propriétés par le Conseil d'administration qui seul aura le droit d'en constater les bons et les mauvais effets par de prudents essais». Ordonnance du Roi concernant les hôpitaux militaires et ceux de charité au compte de Sa Majesté, du $1^{\text {er }}$ janvier 1780, Paris, 1780, article 7, p. 5.

32 Voir par exemple Jean Colombier, Médecine militaire, ou traité des maladies tant internes qu'externes auxquelles les militaires sont exposés dans leurs différentes positions de paix et de guerre, Paris, 1778, p. 389. 
associant les notions fondamentales de médecine, de chirurgie et de pharmacie $^{33}$. Déjà avant la Révolution les Etats accordent de l'importance à la multidisciplinarité et à l'apprentissage des élèves en milieu militaire. C'est ce que nous indique, par exemple, l'ordonnance royale du 20 juillet $1788^{34}$. La nécessité d'un tel apprentissage conduit à la création d'écoles de médecine militaire. L'enseignement se développe d'abord dans les hôpitaux permanents, puis dans les hôpitaux dits d' «instruction». En fait, il a surtout lieu sur le champ de bataille, dans les hôpitaux ambulants et de ligne, c'est-à-dire là où les jeunes officiers de santé peuvent acquérir de l'expérience auprès des grands praticiens. Mais certains hôpitaux militaires autrichiens et français jouissent de la plus grande réputation en tant que centres d'enseignement; par exemple l'Hôtel des Invalides à Paris ou l'Hôpital «Garnisonspital» rattaché à l'Académie Joséphine médico-chirurgicale à Vienne ${ }^{35}$.

Il existe toutefois une certaine inégalité de l'enseignement à l'échelle de l'ensemble des hôpitaux militaires européens. En France notamment, cette inégalité a quelques conséquences négatives sur l'homogénéité de la formation des officiers de santé. C'est pourquoi l'Etat jugera nécessaire de fonder, en 1775, des hôpitaux militaires d'instruction ou hôpitaux-amphithéâtres ${ }^{36}$.Le terme «hôpital-amphithéâtre» vient du règlement du 22 décembre 1775 qui crée les trois hôpitaux d'instruction de Lille, Metz et Strasbourg. Ce règlement stipule qu'il y aura des «amphithéâtres destinés à former en médecine, en chirurgie et en pharmacie des officiers de santé pour le service des hôpitaux militaires du royaume et des armées $»^{37}$. Ces hôpitaux traverseront d'ailleurs une crise quelques années après leur ouverture. Le royaume ne veut

33 Vess, Revolution, pp. 137 sq; Guillermand, Histoire, tome 1er, p. 453; Keel, «Politics», pp. 247 sq. Voir également Dora B. Weiner, «French Doctors Face War: 1792-1815» dans Charles K. Warner (dir.), From the Ancien Régime to the Popular Front: Essays in the History of Modern France, New York \& London, 1969, p. 57.

34 «Lesdits chirurgiens-majors seront à l'avenir choisis de préférence parmi ceux qui auront suivi les écoles établies dans les hôpitaux militaires et qui réuniront les connaissances pratiques et théoriques de la médecine et de la chirurgie; ils seront proposés au Conseil de la guerre après un examen rigoureux sur ces parties, subi par-devant les membres du Conseil de santé des hôpitaux militaires». Ordonnance du Roi portant règlement sur la constitution et l'administration générale des hôpitaux militaires, 20 juillet 1788, Versailles, article 15, pp. 13-14.

35 Guillermand, Histoire, tome $1^{\text {er }}$, p. 455; Baillargeat, Les Invalides; Keel, Cabanis, pp. 404 sq; Keel, «Politics», pp. 242-246; Lesky, Meilensteine, pp. 29-53.

36 Guillermand, Histoire, tome $1^{\text {er }}$, pp. 455-457; Delaunay, Vie médicale, p. 84; Keel, «Politics», p. 247.

37 Règlement fait par ordre du Roi, pour établir dans les hôpitaux militaires de Strasbourg, Metz et Lille, des amphithéâtres destinés à former en médecine, en chirurgie et en pharmacie, des officiers de santé pour le service des hôpitaux militaires du royaume et des armées, 22 décembre 1775. Cité par Jean-Pierre Gama, Esquisse historique du service de santé militaire en général, et spécialement du service chirurgical depuis l'établissement des hôpitaux militaires en France, Paris, 1841, p. 158. 
plus, en effet, limiter l'enseignement de la médecine d'armée aux seuls hôpitaux-amphithéâtres. Il compte l'étendre à tous les grands hôpitaux militaires du pays ${ }^{38}$.

Les hôpitaux d'instruction des armées ont, en moyenne, quatre médecins et quatre chirurgiens chargés d'enseigner les matières médicales et chirurgicales aux futurs praticiens. Avant d'être admis dans un hôpital d'instruction, les élèves chirurgiens doivent déjà avoir deux ans d'apprentissage chez un maître chirurgien. Quant aux élèves médecins, ils sont, tout comme les élèves pharmaciens, tenus d'assister non seulement aux cours de médecine et de pharmacie, mais aussi à ceux de chirurgie. Généralement, le programme d'études des futurs officiers de santé dure trois ans et comprend l'enseignement des trois grandes disciplines; il met en outre l'accent sur la pratique. Les grandes priorités de l'enseignement sont l'anatomie, l'anatomie pathologique, la matière médicale et les principes de la chirurgie. Du reste, les hôpitaux militaires d'instruction ont tous un amphithéâtre d'anatomie et un jardin botanique. Ils représentent, soulignons-le, un apport notable du $18^{\mathrm{e}}$ siècle aux services de santé des grands pays européens.

L'une des caractéristiques les plus novatrices des hôpitaux militaires est sans contredit l'importance accordée à la pratique clinique au lit du malade. En fait, la clinique ne tarde pas à devenir dans les grandes armées de l'Europe un des maillons fondamentaux de la médecine militaire ${ }^{39}$. Il est clair qu'on a le souci de bien encadrer les élèves. L'examen, par exemple, doit sanctionner le succès ou l'échec de l'apprentissage des étudiants. Le rituel clinique doit avoir lieu à une heure bien précise ${ }^{40}$. L'enseignement clinique semble être plus développé en France aux armées que dans les milieux civils. Du moins,

38 Ordonnance du Roi du $1^{\text {er }}$ janvier 1780, article 3, p. 3. «Les trois amphithéâtres créés par le Règlement du 23 [sic] décembre 1775 dans les hôpitaux de Strasbourg, Metz et Lille sont des objets de dépenses superflues» pour ne former qu'un nombre limité d'élèves. «Pour suppléer à la suppression de ces amphithéâtres et former le nombre d'officiers de santé dont les hôpitaux militaires ont besoin tant en paix qu'en guerre, Sa Majesté ordonne que dans chacun de ces hôpitaux, ainsi que dans les établissements de charité à son compte, il soit établi un médecin, un chirurgien et un pharmacien déjà instruits dans la pratique de leur art». Ibid., article 4,p. 4.Voir également Delaunay, Vie médicale, p. 84. A noter que les mesures proposées dans l'ordonnance de 1780 n'ont été que transitoires. En fait, si les hôpitaux-amphithéâtres ont été abolis en 1780, ils ont été rétablis dès mai 1781 avec l'ajout d'hôpitaux nouveaux à Brest et à Toulon. Cf. Ibid., p. 84; Fabre, Histoire, tome 2, p. 13.

39 Bruppacher, Militärmedizin; Lesky, Meilensteine; Moerchel, Militärsanitätswesen; Keel, Cabanis; Garrison, Notes; Delaunay, Vie médicale, pp. 84 sq.

40 Voir par exemple, pour la France, la Lettre des officiers de santé en chef de l'armée aux officiers de santé, Maubeuge, le 24 brumaire de l'an II (14 novembre 1793), signée Lagrésie, chirurgien-consultant, Rosapelly, chirurgien-major, et Renuart, apothicaire-en-chef, pp. 2-3 (Archives du Musée du Val-de-Grâce, Paris, carton 2, dossier 1). Voir plus généralement Guillermand, Histoire, tome $1^{\mathrm{er}}$. Pour la formation des praticiens militaires en Autriche, $c f$. Lesky, Meilensteine, et Moerchel, Militärsanitätswesen. 
c'est chez les militaires qu'il est d'abord organisét1. Déjà au milieu du $18^{\mathrm{e}}$ siècle les hôpitaux des armées préparent le terrain à l'enseignement et à la recherche; en Prusse d'abord, puis en France ${ }^{42}$.

La précocité de la médicalisation et l'émergence d'une médecine clinique en milieu militaire s'expliquent avant tout par le grand nombre et la relative homogénéité des malades, couplés à la spécificité des pathologies des armées. Par exemple, les nombreuses blessures, les maladies dermato-vénériennes, ainsi que les cas très nombreux de maladies infectieuses des systèmes digestifs ou respiratoires permettront aux chirurgiens, puis aux pharmaciens et aux médecins, d'observer et de traiter des organes et des tissus lésés. On en voit des exemples nombreux dans la pratique de médecins et de chirurgiens militaires (ou d'ex-praticiens militaires) comme John Pringle ${ }^{43}$, John Hunter, Donald Monro et bien d'autres. Ainsi, les Observations on the Diseases of the Army de Pringle, recueillies principalement dans les hôpitaux occupés par les troupes britanniques en campagne, attestent une pratique très fréquente de l'inspection cadavérique:

«The liver is a part not only liable to original inflammations, but also to suffer by translations of matter. I have found, by several dissections, that this viscus, next to the lungs, is most subject to suppuration...» ${ }^{44}$.

41 Delaunay, Vie médicale, p. 84; Keel, «Politics», p. 247. A Vienne, cependant, il a été mis en place dans le milieu civil avant de l'être dans le milieu militaire, puis il s'est développé parallèlement dans les deux milieux. Cf. Lesky, Meilensteine; Keel, Cabanis.

42 Voir Keel, «Politics», pp. 233,245,247-248. Par exemple, dès 1724, les instructions de FrédéricGuillaume $1^{\mathrm{er}}$ au Generalchirurgus de son armée lui demandant d'ordonner aux chirurgiens militaires de base que: «In cases of death, he must conduct the post-mortem section with the presence of an officer, handing in his report to the commanding officer». Cité par Garrison, Notes, p. 142.

43 John Pringle (1707-1782), M. D., est le fondateur de la médecine et de l'hygiène militaires modernes. Ses travaux sur la médecine militaire, basés sur une large et longue expérience et sur des recherches systématiques, ont fait autorité dans toute l'Europe et, notamment, en France. Il a d'abord exercé la médecine dans le civil à Edimbourg à partir de 1730. Lors de la guerre de succession d'Autriche (1740-1748), il est nommé, en 1742, médecin de l'hôpital militaire pour les soldats britanniques en Flandre. Il fait la campagne d'Allemagne en 1743. En 1744, il est nommé médecin-général (physician general) de l'armée britannique. Il poursuit sa carrière de physician general de l'armée - un poste de très grande influence - jusqu'en 1758, puis il passe à la pratique civile. Il est nommé médecin de la reine en 1774 et médecin du roi en 1776. En 1772, il avait été nommé président du Royal College of Physicians. Dictionary of National Biography.

44 John Pringle, Observations on the Diseases of the Army, Londres, 1772, $7^{\mathrm{T}}$ édition (1 ${ }^{\mathrm{èr}}$ édition en 1752), p. 145. Souligné par nous. Un nombre important de ces maladies des soldats sont des inflammations de l'estomac et des intestins provoquant la dysenterie ou des coliques. Pringle écrit: «Having described the disease [la dysenterie, NDR], I shall next relate the changes which I have observed in the bodies of those who died of it and were opened».Ibid., p. 237. L'auteur ajoute que: «Sydenham... had never inquired how the bowels of those who died of the distemper [la dysenterie, NDR] appeared after death, nor apprehended any danger from a mortification, which from numerous dissections we are now assured is always threatened». Ibid., p. 149. C'est nous qui soulignons. 
Pringle, sur la base de son expérience, souligne les limites chez Morgagni de sa pratique de l'autopsie des malades décédés des suites de la dysenterie ${ }^{45}$. Autre passage qui montre que l'inspection cadavérique est chez le praticien militaire britannique une pratique courante:

«Not but that among soldiers the bowels are often inflamed; but every inflammation there does not tend to an ileus; for by falling upon the larger intestines it generally occasions a flux, as will appear by the dissections of those who died of the dysentery ${ }^{46}$.»

Pringle affirme qu'il a eu des occasions fréquentes d'examiner la dysenterie sous toutes ses formes et, après avoir présenté la description clinique de cette maladie qui a emporté de nombreux soldats, il donne la relation des altérations anatomopathologiques qu'il a observées. L'examen porte non seulement sur les lésions des organes mais aussi sur celles des tissus. Voici, par exemple, l'observation cadavérique d'un soldat faite en automne 1744 à l'hôpital de Bruxelles:

«I found the larger intestines of a blackish colour and putrid appearance, the coats preternaturally thick (the mark of a preceding inflammation) and on the inside ulcerated, especially in the rectum and lower part of the colon, where the villous coat was either abraded, or changed into a corrupted slimy substance of a greenish cast. The caecum and its appendix were less tainted; and the smaller intestines and stomach were neither mortified, discoloured, nor abraded, but only distended with $\operatorname{air}^{47}$.»

Autre cas, celui d'un soldat d'artillerie, dans le même hôpital, au cours de la même saison:

«The rectum was most putrid; and from thence the gangrene seemed to have spread itself to the colon, which was mortified, and chiefly at its lower end. The villous coat was partly consumed, and that remained was blackish, tender and easily to be separated. The vascular coat had the appearance of a preparation well injected with red wax. The ligaments, which contract the colon and form the cells, were half corrupted, and adhered loosely to the outer $\underline{\operatorname{coat}}^{48} \cdot{ }^{\prime \prime}$

45 «For though this excellent anatomist has, in his usual manner, made some useful remarks upon the disease, and has added a few dissections of his own, yet as his cases seem to have been all of the sporadic kind, I must consider them as somewhat different from such as we are now treating of. Indeed Morgagni acquaints us, that he had generally declined opening the bodies of those who died of any infectious distemper». Ibid., p. 238. Souligné par nous. L'ouvrage de Morgagni cité est le De sedibus et causis morborum per anatomen indagatis (1761). Comme on va le voir, les médecins militaires tel Pringle font avancer, dans la seconde moitié du $18^{\mathrm{e}}$ siècle, la médecine anatomoclinique en complétant les résultats de Morgagni et en situant la lésion non seulement au niveau de l'organe, mais encore à celui du tissu.

46 Ibid., p. 153. Plus loin, il ajoute: «Obstructions of the lungs are succeded by tubercles and ulcerations. In several bodies which I examined of those who died of the phtisis pulmonalis, I found the lungs adhering to the pleura, and full both of tubercles and ulcers». Ibid., p. 163. Souligné par nous.

47 Ibid., p. 239. Souligné par nous.

48 Ibid., p. 240. Pringle mentionne deux autres cas de soldats atteints de cette dysenterie épidémique décédés dans ce même hôpital pendant la même saison et où il a examiné les lésions des organes et des tissus. Voici un autre exemple des observations histopathologiques qu'il a consigné dans ces cas: «Although the body was opened the next day, yet the smell was 
Pringle mentionne aussi une inspection cadavérique d'un malade décédé d'une dysenterie qu'il a faite dans le milieu civil à Londres. Le cas qui suit présente un intérêt particulier pour plusieurs raisons. Premièrement, il s'agit d'observations anatomo-cliniques faites dans la pratique privée et non à l'hôpital. A ce moment-là, Pringle exerce dans le milieu civil, qu'il fait profiter de son expérience acquise dans la pratique militaire. La dissection et l'inspection cadavérique ont été faites en automne 1762, le jour suivant le décès du patient et à son domicile où Pringle avait été appelé au début de la maladie. Les traitements tentés s'étaient avérés vains. Deuxièmement, Pringle a procédé en collaboration avec un chirurgien, «Mr Hewson» et un médecin, le «Dr Huck». L'ouverture du corps a été faite par le chirurgien Hewson, mais l'inspection cadavérique a été faite de concert par Pringle, Hewson et le Dr Huck ${ }^{49}$. Troisièmement, on peut noter que les praticiens ou ex-praticiens militaires et ceux du milieu civil peuvent collaborer étroitement, ce qui permet des échanges fructueux de leur expérience clinique respective. Quatrièmement, on voit sur ce cas aussi que ces praticiens examinent déjà les lésions au niveau tissulaire et qu'ils poussent l'examen en profondeur pour déterminer la nature de la lésion, son étendue et la couche tissulaire principalement affectée. On confronte les observations recueillies avec des pièces anatomopathologiques de cas précédents, ou avec les observations de cas semblables faites par des praticiens militaires ou civils:

«On the day after his death, the body was opened by Mr Hewson, surgeon and anatomist, Dr Huck and I being present. Upon cutting into the abdomen, we found the tunica adiposa of a considerable thickness, notwithstanding the long continuance of the disease. The stomach and smaller intestines were inflated, but otherwise in a natural state, except at the extremity of the ileum where it joins the caecum; for at that place the coats of the gut were thicker and more tender than they ought to have been; and on the inside we found that glossy colour which is considered as a mark of inflammation ${ }^{50} . »$

intolerable. The intestines seemed mortified. The outward coat of the liver was putrid; and in the substance of that viscus we found several abcesses containing a purulent or ichorous matter. The spleen was likewise corrupted; but the kidneys, heart and lungs appeared to be sound». Ibid., p. 242. «In an external gangrene, we commonly observe some vesicles of air in the cellular membrane, which vesicles being wanting in those subjects, it may be disputed whether the mortification of the bowels was complete without them». Ibid., p. 248. Soulignés de nous.

49 Il était habituel à Londres, dans les hôpitaux comme dans la pratique privée, que les médecins fassent appel à un de leurs collègues chirurgiens pour ouvrir le corps d'un patient dont ils voulaient faire l'inspection anatomopathologique. Alors, le plus souvent, cette inspection était faite à la fois par le médecin et le chirurgien qui examinaient ensemble les lésions et les corrélations avec les symptômes et signes cliniques qui avaient précédé, et qui discutaient ainsi des cas. Souvent, on faisait aussi appel à un autre médecin et/ou à un autre chirurgien pour faire conjointement l'inspection nécroscopique et discuter ainsi à trois ou à quatre du "cas anatomoclinique».

50 Ibid., p. 244. L'exposé de l'examen à plusieurs du cas anatomoclinique se poursuit ainsi: "At first sight the villous coat seemed to have been resolved into the slime above mentioned, yet 


\section{On trouve aussi de nombreux exemples d'une pratique anatomoclinique semblable chez d'autres médecins ou chirurgiens militaires; à preuve, Donald Monro, M.D. (1727-1802):}

«The appearances we found after death in the bodies of some patients, who died of old fluxes at Bremen, were: in all of them the rectum was inflamed, and partly gangrened, especially the internal coat. In two the lower part of the colon was inflamed, and there were several livid spots on its great arcade. In one whose body was much emaciated, and who had been seized with a violent pain of the bowels two days before his death, all the small guts were red and inflamed; and in another there were livid gangrened spots on the stomach ${ }^{51}$.»

upon a nearer inspection we thought it more probable, that both in the rectum and colon that coat, though diseased, yet was not separated, whatever it might be in the rectum, which was too putrid to be minutely examined". (Ibid., p. 245). "Mr Hewson was of opinion that they [les tubercules, NDR] took their rise from the cellular membrane which lies immediately above the villous coat; for that some days before, having opened another person, who had likewise died of the dysentery, he had found the appearances there much the same as in this subject, and particularly with regard to these tubercles, which he had examined at leisure. He added that he had preserved a part of the colon in spirits, which he would shew us some other time". (Ibid., p. 246). "Some time afterwards, Mr Hewson shewed me that portion of the colon, which he had cut out of the other body and had preserved in spirits; he said that to the best of his remembrance he had taken it from the lower part of the gut. I could easily trace the resemblance between this preparation and what $\mathrm{I}$ had seen in the recent subject, though the tubercles were here more numerous, and generally higher than in the other. Dr Hunter, who was present, did not recollect to have seen that kind of morbid appearance before, but was satisfied that the villous coat had not been separated, further than some partial abrasions might have been seen on dissection. (Ibid., pp. 247-248). "And Mr Cleghorn, who had frequent opportunities, at Minorca, of seeing the epidemic dysentery, observes: 'That upon opening the bodies, he constantly found the great guts either entirely mortified, or partly inflamed, partly mortified; that the rectum was most affected; and that in many he had seen scirrhous tubercles straitening the cavity of the colon in several places'. Although those tubercles, which I have described in my patient were too flat to be taken notice of, as straitening the cavity, yet in Mr Hewson's preparation they were perhaps large enough to have had that effect". (Ibid., p. 249. L'ouvrage de George Cleghorn cité est ses Observations on the Epidemical Disease in Minorca from the Year 1744 to 1749, London, 1751). "In the first dissection I mention the abrasion of the villous coat, and perhaps in my account of the last, I should have made the same observation, had not Mr Hewson been inclined to think otherwise; and had not Dr Hunter, in viewing the preparation (which was mentioned before) been of opinion that in this portion of the gut, the villous coat had not been separated, though perhaps fissured, and a little abraded on the top of some of the tubercles; and that, from the account which Mr Hewson and I had given him of the last subject, there had been no considerable abrasion in those intestines, more than in this piece which was before him". (Ibid., p. 250). Les soulignés sont de nous.

51 Donald Monro, An Account of the Diseases which were most frequent in the British military hospitals in Germany, from january 1761 to the return of the Troops to England in march 1763, London, 1764, pp. 63-64. Souligné par nous. Le médecin ajoute en note: "From the accounts we have in authors of the dissection of the bodies of persons who died of the dysentery, it would appear that there is no part of the alimentary canal which has not some time or other been found inflamed, or in a state of suppuration or gangrene; and the liver, spleen, and other viscera, have likewise been found diseased, but the rectum and colon have almost in all been more or less affected. (...) While this sheet was in the press, I received Dr Pringle's $4^{\text {th }}$ edition of his Observations on the diseases of the army, and Dr Baker's treatise on the Dysentery which was epidemic in London in the year 1762. Both these gentlemen give an account of the dissection of the bodies of some people who died of the dysentery; where, besides the 


\title{
Sur la péripneumonie, Monro affirme:
}

«In most of the bodies of those who died of this disorder, and were opened after death, we found the lungs violently inflamed, with livid or gangrenous spots on their surface; and more or less of a watery serum extravasated into the cavity of the chest ${ }^{52}$.»

\section{Plus loin, il note que:}

"On examining, I observed a fullness in that side of the thorax, and on pressing with my fingers between the ribs, I thought I felt an obscure fluctuation of a deep-seated fluid. From these appearances and the history of the case, I judged that there was a collection of some fluid within the cavity of the chest; and that the only means left to give relief was to make an opening into the cavity, and so evacuate the fluid. (...) The pericardium was thickened and slightly inflamed; and adhered to the diaphragm, which was likewise a little thickened and inflamed in the adhering part. The lungs on that side were much compressed and contracted by the pressure of the water; but on being inflated and cut, seemed in a sound state, except that they were slightly inflamed ${ }^{53} . »$

On notera l'importance de l'examen physique (palpation, etc.) dans le diagnostic des maladies internes, et cela en vue d'une intervention chirurgicale (la ponction thoracique, par exemple) chez les médecins militaires comme Donald Monro, qui ajoute, à propos de la péripneumonie et de la pleurésie:

\begin{abstract}
«Some late authors seem to look upon the pleurisy and peripneumony as the same disorder; however, though it be true that when the pleura is inflamed, the surface of the contiguous lungs is generally in the same state; and that when the lungs are inflamed, the pleura is often affected; yet as I have frequently seen the true peripneumony without that sharp pain of the side which characterizes the pleurisy; and upon opening the bodies of people who have died of the peripneumony, have found the lungs violently inflamed and livid, and so filled with blood as to sink in water, without the pleura being much diseased; and on upon opening the thorax of others who died of the pleurisy, have found the intercostal muscles and pleura violently inflamed with livid spots, and only a small portion of the surface of the contiguous lungs affected. I cannot help still looking upon them as distinct disorders, though they require nearly the same treatment, and are often complicated together ${ }^{54} . »$
\end{abstract}

On voit ici que, bien avant Bichat, l'auteur a très bien observé que la lésion a pour siège l'un des tissus constituants de l'organe affecté et non pas l'organe dans son ensemble et que, alors que ce tissu est altéré, les autres tissus

common appearances of the inner surface of the rectum and colon being covered with a bloody slime, and their internal coats being inflamed, gangrened, or in a putrid state, there were observed on the inside of the lower part of the colon and upper part of the rectum a number of little tubercles, or excrescences, which resembled the small pox, of a flat sort at the height of the disorder; but differed from them in this, that they were of a firm consistence, without any cavity: they were believed to take their rise from the cellular membrane, which lies immediately above the villous coat.Ibid.,pp.64-65.Plus loin,Monro indique:"On opening his body, the inner coats of the rectum and the lower part of the colon seemed to be reduced almost to a gelatinous substance, and the other coats were black, approaching to a gangrene". Ibid., p. 87. C'est nous qui soulignons.

52 Ibid., p. 116.

53 Ibid., pp. 119-120. C'est nous qui soulignons.

54 Ibid., p. 123. Souligné par nous. 
constituants peuvent demeurer sains (au moins en partie). Ce principe anatomopathologique combiné avec l'usage de moyens physiques d'examen, permet d'établir un diagnostic différentiel entre la pleurésie et la péripneumonie ${ }^{55}$.

Le célèbre John Hunter (1728-1793), chirurgien britannique, a exercé son métier dans le service médical des armées de sa Majesté de 1761 à 1763, notamment lors de l'expédition de Belle-Ile (1761) et pendant la guerre au Portugal $(1762)^{56}$. Il a consigné quelques-unes de ses observations anatomopathologiques sur des militaires dans son Traité sur le sang, l'inflammation et les plaies d'armes à feu (1794). A propos de l'inflammation qui résulte d'une blessure causée par des projectiles d'armes à feu, il note que:

«But although the ball has passed with such velocity as to produce a slough, yet that wound shall do well, for the adhesive inflammation will take place on the peritoneum all round the wound, which will exclude the general cavity from taking part in the inflammation, although the ball has not only penetrated but has wounded parts which are not immediately essential to life, such as the epiploon, mesentery, etc., and perhaps gone quite through the body; yet it is observed that wherever there is a wound, and whatever solid viscus may be penetrated, the surfaces in contact surrounding every orifice will unite by the adhesive inflammation, so as to exclude entirely the general cavity, by which means there is one continued canal wherever the ball or instrument has passed; or if any extraneous body should have been carried in, such as clothes, etc., they will also be included in these adhesions, and both these and the slough will be conducted to the external surface by either orifice ${ }^{57}$.»

En France, la correspondance des praticiens militaires exerçant au front, adressée aux chefs des services de santé des armées, constitue une source très intéressante d'observations anatomopathologiques. Par exemple, une lettre d'un médecin dénommé Bagneris, attaché à l'hôpital sédentaire de Listroff dans les premiers temps des guerres de la Révolution. Bagneris affirme que l'hôpital a reçu un grand nombre de soldats affligés d'affections catarrhales

55 Pour d'autres exemples d'une telle pratique anatomoclinique et histopathologique courante en Europe, et notamment en Angleterre bien avant l'École clinique de Paris, $c f$. Keel, $L a$ généalogie de l'histopathologie. Une révision déchirante: Philippe Pinel, lecteur discret de J. C. Smyth (1741-1821), Paris, 1979; Idem, «Percussion et diagnostic physique en GrandeBretagne au $18^{\mathrm{e}}$ siècle: l'exemple d'Alexander Monro secundus», dans Actes du $31^{e}$ Congrès international d'histoire de la médecine, Bologne 1988, Bologne, 1988, pp. 869-875.

56 C'est à Belle-Ile que Hunter a acquis une connaissance approfondie des plaies par armes à feu. En 1764, Hunter revient à Londres comme demi-solde. Il exerce alors dans le civil. En 1786, il est full pay, assistant surgeon-general et en 1790 il est surgeon-general, puis inspector of regimental hospitals. Voir dans Dictionary of National Biography.

57 John Hunter, A Treatise on the Blood, Inflammation and Gunshot Wounds, dans The Complete Works of John Hunter, F.R.S., édité par James Palmer, vol. 3, Philadelphia, 1841, pp. 525-526. Les soulignés sont de nous. Hunter a aussi, avant l'École clinique de Paris, observé systématiquement que la lésion peut affecter un tissu (ici le péritoine) à l'exclusion des autres tissus constituants d'un organe ou d'une partie de l'organe. $C f$. sur ce point Keel, «John Hunter et Xavier Bichat: Les rapports de leurs travaux en pathologie tissulaire» dans Actes du $27^{e}$ Congrès International d'histoire de la médecine, Barcelone 1980, Barcelone, 1981,pp.535-549. Voir aussi Hunter, Treatise, pp. 534-535, au sujet des observations anatomopathologiques sur des soldats décédés de blessures à la poitrine par armes blanches. 
qui sont par la suite décédés. Il pratique systématiquement la dissection de ces militaires morts ${ }^{58}$.

Les médecins et chirurgiens militaires, on le voit, sont tout naturellement confrontés à une pathologie organique; et cette approche anatomopathologique, qui se fait par le biais de la clinique et grâce à une nouvelle compréhension des concepts médicaux, rend possible l'avancement des connaissances générales en chirurgie comme en médecine et en pharmacie. En particulier, ces nouvelles connaissances permettent de localiser la lésion au niveau de l'organe et même des tissus (membrane interne, ou muqueuse de l'intestin). La médecine aux armées favorise donc au $18^{\mathrm{e}}$ siècle, comme d'ailleurs la médecine civile dans certains secteurs et parfois par osmose entre les deux, le développement d'une clinique anatomique et d'une pathologie localiste (au niveau non seulement des organes, mais encore des tissus). Il va de soi que la précondition de ce développement est une pratique déjà importante de la dissection et d'un examen physique (palpation, etc.) des malades $^{59}$. Davantage que le médecin civil, le chirurgien ou le médecin militaire a l'opportunité de «voir» à l'intérieur du malade ${ }^{60}$. Cette approche clinique anatomo-localiste est d'ailleurs bien mise en évidence chez les auteurs cités ${ }^{61}$. Ce fait revêt une importance extrême dans la mesure où les médecins et chirurgiens militaires, qu'ils soient dans les hôpitaux d'instruction, les hôpitaux spécialisés ou au front, forment de cette façon des centaines d'élèves qui, comme eux, interviennent de plus en plus au nom de ces nouvelles connais-

58 «L'ouverture de leur cadavre, dit-il, à laquelle j'ai veillé avec le plus grand soin, m'a toujours fait voir l'un des poumons, et quelquefois tous les deux, fortement enflammés. Ils étaient d'un rouge noir et d'un volume considérables. Les sujets chez qui la maladie avait duré plusieurs jours avaient des points de suppuration répandus dans la substance des poumons. J'ai trouvé dans la poitrine de deux soldats le poumon gauche presque détruit, avec une quantité considérable de pus dans la cavité du même côté». Bagneris, Observations du médecin de l'hôpital sédentaire de Listroff, à la suite de l'armée de la Moselle, sur les maladies régnantes, Listroff, le 31 janvier 1792 (ou 1793), pp. 1-2. Plus loin dans sa lettre, Bagneris affirme avoir trouvé dans le corps d'un autre soldat décédé: «... la plèvre, les poumons, le péricarde et le cœur dans l'état de la plus grande inflammation. Une couenne inflammatoire très épaisse recouvrait toutes les parties. Le foie était aussi atteint d'inflammation». Ibid., p. 6.

59 Pour l'approche anatomolocaliste chez les chirurgiens et les médecins de la seconde moitié du $18^{\mathrm{e}}$ siècle, voir Owsei Temkin, «The Role of Surgery in the Rise of Modern Medical Thought» dans The Double Face of Janus and Other Essays in the History of Medicine, Baltimore \& London, 1977; Keel, Cabanis; Idem, «La pathologie tissulaire de John Hunter» dans Gesnerus, 37, 1980, pp. 47-61; Idem, «Les conditions de la décomposition 〈analytique〉 de l'organisme: Haller, Hunter, Bichat» dans Les Etudes Philosophiques, 1, 1982, pp. 37-62; Idem, «Les rapports», pp. 323-341; Imbault-Huart, «Les chirurgiens», pp. 143-157; Christopher Lawrence (dir.), Medical Theory, Surgical Practice. Studies in the History of Surgery, London \& New York, 1992; voir enfin Toby Gelfand, Professionalizing Modern Medicine. Paris Surgeons and Medical Science and Institutions in the $18^{\text {th }}$ Century, Westport \& London, 1980.

60 Temkin, «Role of Surgery». Mais cela est également vrai pour un nombre important de médecins civils. Voir Keel, Cabanis; Idem, Généalogie; Idem, «Percussion». 
sances anatomopathologiques. Ces mêmes élèves formeront à leur tour de nouveaux praticiens et affineront ces concepts.

Ces observations et ce nouveau mode d'examen clinique sont favorisés par des conditions propres au monde militaire:l'homogénéité de l'âge et du sexe, la force physique et surtout la discipline militaire, qui est aussi sévèrement appliquée dans les hôpitaux que dans les camps. En somme, l'affirmation, l'extension et le maintien du pouvoir des états-majors sur les hommes sont imposés à partir de critères sanitaires et prophylactiques dans la réalisation desquels les médecins, les pharmaciens et les chirurgiens des armées sont appelés à jouer un rôle primordial. Il s'agit alors de tenir sur les soldats malades un discours à structure plus «scientifique», plus médicale, autorisant l'application d'une discipline «nouvelle». Dans cette optique, l'hôpital militaire devient un des lieux privilégiés de l'exercice de ce qu'on a appelé la «biopolitique ${ }^{62}$. Ceci s'inscrit dans le cadre de la mise en place, au cours du $18^{\mathrm{e}}$ siècle dans les grands pays européens, de nouvelles politiques de croissance démographique inspirées par les doctrines populationnistes ${ }^{63}$, au sein desquelles les hôpitaux (y compris les hôpitaux militaires) ont un rôle central.

C'est dans un tel contexte que se produit l'essor de l'enseignement clinique. Les praticiens militaires ont souvent l'obligation de donner un enseignement dit «clinique», c'est-à-dire d'instruire les élèves, futurs médecins ou chirurgiens des armées, sur la façon de reconnaître, d'observer et de traiter les maladies des soldats qui se présentent en très grand nombre dans les hôpitaux. Cet enseignement a lieu au chevet des militaires hospitalisés ou admis sous les tentes destinées à procurer des soins thérapeutiques ${ }^{64}$. Tous les types

61 Une telle pratique est représentative d'une médecine antérieure à celle de l'Ecole clinique de Paris, y compris en France même. Laribeau par exemple, qui s'intéresse à la gale, va jusqu'à provoquer des lésions cutanées pour mieux étudier les conditions d'infection de la gale. $\mathrm{Ce}$ chirurgien touche et observe, il intervient. Cette approche anatomoclinique va permettre à la chirurgie, puis à la médecine toute entière, de franchir en quelques décennies un grand pas dans les connaissances des symptômes cliniques, et ce malgré les incertitudes qui subsistent à propos des agents qui causent la gale. $C f$. Laribeau, Mémoire sur la gale présenté à la Commission de Santé, le 27 messidor de l'an II (15 juillet 1794), sans nom de lieu, manuscrit, (Archives du Musée du Val-de-Grâce, Paris, Carton 2, Dossier 1).

62 Cf. Foucault, Surveiller, pp. 197-229, et Keel, Cabanis.

63 Le populationnisme, dans ses variantes mercantiliste et caméraliste, voit le jour dans différents pays européens dans le courant du $17^{\mathrm{e}}$ siècle et s'affirme surtout au siècle suivant. C'est le cas de l'Autriche, de la Prusse, de l'Italie, de l'Angleterre et de la France. $C f$. Keel, «Politics»,pp.214-217; George Rosen, From Medical Police to Social Medicine. Essays on the History of Health Care, New York, 1974.

64 Nous avons, dans un article récent, souligné toute l'importance du transfert des soldats vénériens des hôpitaux permanents éloignés du front vers les tentes des régiments situées sur la ligne de feu. Ce transfert, conçu et dirigé par les officiers de santé exerçant sur le terrain, a rapidement mis en évidence des avantages que ne possèdent pas les hôpitaux militaires traditionnels. $C f$. Philippe Hudon, «Les soldats vénériens pendant la Révolution et le Premier Empire. Les défis de la médicalisation» dans Cahiers du Centre de recherches histo- 
de patients sont observés et ce en très grand nombre. L'exercice d'une médecine clinique ainsi structurée a lieu, soulignons-le, bien avant la Révolution dans la plupart des armées européennes. Déjà au milieu du siècle les ordonnances royales exhortent les praticiens militaires à fournir à leurs élèves des cours de médecine clinique. En France, notamment,l'ordonnance de 1747 enjoint aux médecins et aux chirurgiens des armées d'enseigner de cette manière. Le règlement de 1775 va réitérer cet ordre ${ }^{65}$. En France, en Prusse et en Autriche, l'Etat veut faire des hôpitaux dits d'instruction de «véritables cliniques militaires» ${ }^{6}$. Il faut souligner, en outre, que les élèves en clinique feront souvent l'objet d'une étroite surveillance.

La médicalisation des armées a lieu, au $18^{\mathrm{e}}$ siècle, parfois en parallèle avec la médicalisation en milieu civil, parfois en avance sur elle. Elle aura donné des résultats positifs et même apporté de nouvelles connaissances scientifiques et techniques à la médecine en permettant des avancées dans la clinique et la pathologie organique et tissulaire. La guerre, notamment, aura favorisé ces progrès importants et assuré la diffusion d'une vague d'informations et de statistiques cliniques à partir des hôpitaux militaires vers tous les autres centres hospitaliers, qu'ils soient militaires, civils ou maritimes, et ce dans toute l'Europe ${ }^{67}$. La tenue d'une information statistique, déjà préconisée avant la Révolution ${ }^{68}$, est un des corollaires de la mise en place de l'exercice d'une biopolitique et constitue une des principales ressources de la nouvelle médecine.

Ce processus de médicalisation, observable dans toutes les grandes puissances européennes du $18^{\mathrm{e}}$ siècle, constitue un des maillons fondamentaux assurant l'avant-gardisme de la médecine militaire. Les médecins, pharmaciens et chirurgiens qui se portent au secours des soldats malades font désormais partie d'un vaste cadre médical et hospitalier qui se développe, se diversifie et se complexifie. Ils opèrent dans des hôpitaux exclusivement

riques, $\mathrm{n}^{\circ} 12$ thématique: «Polysémie de la santé. Institutions et pratiques sociales en France et au Québec», édité par Jean-Pierre Goubert et Othmar Keel, Centre de Recherches Historiques, EHESS et CNRS, Paris, 1994, pp. 13-31.

65 «Conformément au titre VII, article $1^{\text {er }}$ de l'Ordonnance du $1^{\text {er }}$ janvier 1747 , il y aura cours de physiologie, de pathologie et en même temps un cours de pratique et clinique des principales maladies qui règnent parmi les troupes dans les armées et les garnisons». Règlement $d u$ 22 décembre 1775 cité par Gama, Esquisse, p. 158.

66 Pour la France, voir Léon Mention, L'armée de l'Ancien Régime, Paris, 1900, p. 277. Pour l'Autriche, se référer à Moerchel, Militärsanitätswesen, p. 138 sq.; Keel, Cabanis, p. 475 sq.

67 Vess, Revolution, pp. 146 sq.

68 En France,l'ordonnance de 1780 stipule: «Ordonne Sa Majesté aux Commissaires des guerres et subdélégués chargés de la police des hôpitaux d'écrire sur le dos des billets de sortie qui seront expédiés aux soldats, cavaliers ou dragons guéris de maux vénériens la nature de chaque maladie et le nombre de jours qui aura été employé à leur guérison». Code d'administration des hôpitaux militaires et de charité, au compte du Roi, Paris, $1^{\text {er }}$ janvier 1780 , article 5, p. 103. Ce code d'administration est annexé à l'Ordonnance de 1780 déjà citée. 
destinés aux militaires qui, avant même les guerres de la Révolution, se sont multipliés un peu partout. Ces hôpitaux et leur personnel rendent possible la création et la diffusion d'un nouveau savoir médical à partir des nombreuses observations de cas pathologiques qui s'y font. La diffusion de ce nouveau savoir accentue les échanges entre les institutions médicales militaires tout en offrant les conditions propices à leur médicalisation: création d'hôpitaux spéciaux ou d'instruction, observation clinique plus stricte des malades, tenue de statistiques sur le nombre des admissions et des cas pathologiques, dissections, etc. ${ }^{69}$.Par conséquent, les connaissances ainsi acquises par les praticiens des armées vont pouvoir être utilisées au profit non seulement de la médecine militaire mais, au bout du compte, de l'ensemble de la médecine.

69 En Grande-Bretagne, un service de santé militaire régulier permanent avait été institué avec la mise en place de la «Standing Army» à partir de 1660 pour l'armée de terre et, pour la marine, à partir de la création du Sick and Hurt Board (1653). On assiste là aussi à un lent et difficile processus de médicalisation des armées, mais qui produit à la longue un meilleur encadrement sanitaire et médical des soldats. Le milieu des armées permet l'essor d'une pratique et d'une formation cliniques, même si cette formation est informelle et se fait «sur le tas». En effet, si, pour l'armée de terre, la grande majorité des hôpitaux fonctionnent de façon temporaire, notamment ceux qui sont ouverts sur le Continent, le personnel médical des regimental infirmaries est plus stable; pour ces dernières comme pour les general hospitals, le personnel d'encadrement (y compris les mates et/ou les nurses) a tendance à augmenter. L'ouverture et la fermeture des general hospitals selon les aléas de la situation militaire pouvait être très pénalisante, en raison de la désorganisation au moment de la reprise des hostilités, mais il y avait cependant une amélioration des soins assurés et dispensés au cours de la progression des guerres. La politique des autorités était d'ailleurs de ne recourir qu'en dernière instance au general hospital, à cause des risques élevés d'épidémies et de s'appuyer plutôt, même si le niveau du personnel y était plutôt bas, sur les structures plus «légères» des regimental infirmaries qui étaient tout de même un terrain d'expérience et de formation. On constate même une amélioration des services rendus aux patients par les hôpitaux et une baisse marquée de la mortalité dans ces derniers, par exemple, au cours de la guerre de Succession d'Autriche (baisse de plus de $10 \%$ entre 1742 et 1748). Le service de santé de la marine dispose, lui, de grands hôpitaux permanents qui avec les navires-hôpitaux et les vaisseaux de la flotte servent aussi de terrain d'expérience, de recherche, et de formation (de manière informelle). Les insuffisances des services de santé militaires britanniques étaient dues à la parcimonie du Trésor public, et par suite, du Secrétariat à la Guerre. Les soldes, très pauvres, rendaient difficile le recrutement d'un personnel compétent qui devait donc parfaire sa formation «sur le tas». C'est aussi ce qui explique l'insuffisance des hôpitaux militaires permanents pour l'armée de terre avant la fin du $18^{\mathrm{e}}$ siècle, ainsi que l'absence d'hôpitaux militaires d'instruction et de vraies écoles militaires de santé. Malgré tout cela, le service militaire de santé britannique, notamment au niveau de la compétence de son personnel médical, a été considéré comme mieux organisé et plus performant que celui d'autres pays européens comme la Prusse. Cf. Lloyd et Coulter, Medicine and the Navy; Cantlie, History; Troehler, Quantification; Kopperman, «Medical Services». 